\title{
Numerical modelling of foam Couette flows
}

\author{
I. Cheddadi ${ }^{1}$, P. Saramito ${ }^{1, a}$, C. Raufaste ${ }^{2, b}$, P. Marmottant ${ }^{2}$, and F. Graner ${ }^{2, c}$ \\ 1 Laboratoire Jean Kuntzmann, Université J. Fourier, CNRS and INRIA, 51, rue des Mathématiques, 38400 Saint-Martin \\ d'Hères Cedex, France \\ 2 Laboratoire Spectrométrie Physique, UMR 5588, CNRS and Université J. Fourier, B.P. 87, 38402 Saint-Martin d'Hères Cedex, \\ France
}

Original article: Eur. Phys. J. E 27, 123 (2008) DOI: 10.1140/epje/i2008-10358-7

Received: 3 February 2009

Published online: 14 March 2009 - (c) EDP Sciences / Società Italiana di Fisica / Springer-Verlag 2009

We have found a technical error in Appendix A of [1] which induced errors in Appendix B and in the figures. However, the text of the paper, including all conclusions drawn, is completly unaffected by the changes. In [1], equations (A.1a), (A.1b), and (A.1c) should read

$$
\begin{gathered}
\lambda \frac{\partial \tau_{r r}}{\partial t}+\max \left(0,1-\frac{\tau_{Y}}{\left|\boldsymbol{\tau}_{d}\right|}\right) \tau_{r r}=0, \\
\lambda\left(\frac{\partial \tau_{r \theta}}{\partial t}-2 \dot{\epsilon}_{r \theta} \tau_{r r}\right)+\max \left(0,1-\frac{\tau_{Y}}{\left|\boldsymbol{\tau}_{d}\right|}\right) \tau_{r \theta}=2 \eta \dot{\epsilon}_{r \theta},
\end{gathered}
$$

$\lambda\left(\frac{\partial \tau_{\theta \theta}}{\partial t}-4 \dot{\epsilon}_{r \theta} \tau_{r \theta}\right)+\max \left(0,1-\frac{\tau_{Y}}{\left|\boldsymbol{\tau}_{d}\right|}\right) \tau_{\theta \theta}=0$,

with $\dot{\epsilon}_{r \theta}=1 / 2\left(\frac{\partial v}{\partial r}-\frac{v}{r}\right)$. Consequently, Appendix B is modified but its conclusion remains the same: if the strain rate is discontinuous, we can predict the critical strain rate $\dot{\varepsilon}_{r \theta}^{c}=\frac{1}{2 \eta}\left[1-\frac{\tau_{Y}}{\sqrt{2}\left|\tau_{r \theta}\left(r_{c}\right)\right|} \frac{1}{\left(1+\frac{\lambda^{2}}{\eta^{2}} \tau_{r \theta}\left(r_{c}\right)^{2}\right)^{1 / 2}}\right] \tau_{r \theta}\left(r_{c}\right)$.

All the presented computations have been made again,

\footnotetext{
a e-mail: pierre.saramito@imag.fr

b Present address: Physics of Geological Processes, University of Oslo, Sem Selands vei 24, NO-0316 Oslo, Norway.

c Present address: CNRS UMR 3215, Institut Curie, 26 rue d'Ulm, 75248 Paris Cedex 05, France.
}

and most figures are unchanged, except Figures 7, 8(b), and $9(\mathrm{~b})$, whose new versions are shown in the following page.

\section{Modified Appendix B}

[...] We write the constitutive equation with $\frac{\partial}{\partial t}=0$. We have:

- When $r>r_{c}$ : the plasticity term is zero, so (A.1c) leads to $2 \lambda \dot{\epsilon}_{r \theta} \tau_{r \theta}=0$, and $\dot{\epsilon}_{r \theta}=0$ as $\tau_{r \theta} \neq 0$; in addition, we have $v=0$ because of boundary conditions. However, (A.1a) and (A.1b) are then equivalent to $0=0$, and the normal stress components are not determined by the stationary equations only. In the transient problem, their values are determined by the initial conditions. Finally, as $v=0,(\mathrm{~A} .2 \mathrm{~b})$ yields $\tau_{r \theta}=C / r^{2}$, where $C$ is a constant.

- When $r<r_{c}$ : (A.1a, A.1b) lead to

$$
\begin{aligned}
& \left(1-\frac{\tau_{Y}}{\left|\boldsymbol{\tau}_{d}\right|}\right) \tau_{r r}=0 \\
& -2 \lambda \dot{\epsilon}_{r \theta} \tau_{r r}+\left(1-\frac{\tau_{Y}}{\left|\boldsymbol{\tau}_{d}\right|}\right) \tau_{r \theta}=2 \eta \dot{\epsilon}_{r \theta}, \\
& -4 \lambda \dot{\epsilon}_{r \theta} \tau_{r \theta}+\left(1-\frac{\tau_{Y}}{\left|\boldsymbol{\tau}_{d}\right|}\right) \tau_{\theta \theta}=0 .
\end{aligned}
$$

We denote with $a^{-}$(respectively, $a^{+}$) the quantities evaluated in $r=r_{c}^{-}$(respectively in $r=r_{c}^{+}$); $v$ and $\tau_{r \theta}$ are continuous, thus $v^{-}=v^{+}=0, \tau_{r \theta}^{-}=\tau_{r \theta}^{+}=\tau_{r \theta}\left(r_{c}\right)$ and we 
have

$$
\begin{aligned}
& \left(1-\frac{\tau_{Y}}{\left|\boldsymbol{\tau}_{d}^{-}\right|}\right) \tau_{r r}^{-}=0, \\
& -2 \lambda \dot{\epsilon}_{r \theta}^{-} \tau_{r r}^{-}+\left(1-\frac{\tau_{Y}}{\left|\boldsymbol{\tau}_{d}^{-}\right|}\right) \tau_{r \theta}\left(r_{c}\right)=2 \eta \dot{\epsilon}_{r \theta}^{-}, \\
& -4 \lambda \dot{\epsilon}_{r \theta}^{-} \tau_{r \theta}\left(r_{c}\right)+\left(1-\frac{\tau_{Y}}{\left|\tau_{d}^{-}\right|}\right) \tau_{\theta \theta}^{-}=0 .
\end{aligned}
$$

If $1-\frac{\tau_{Y}}{\left|\tau_{d}^{-}\right|}=0$, we find $\dot{\epsilon}_{r \theta}^{-}=0=\dot{\epsilon}_{r \theta}^{+}$: there is no discontinuity.

If $1-\frac{\tau_{Y}}{\left|\boldsymbol{\tau}_{d}^{-}\right|} \neq 0$, we find

$$
\begin{aligned}
\tau_{r r}^{-} & =0 \\
\tau_{\theta \theta}^{-} & =2 \frac{\lambda}{\eta} \tau_{r \theta}\left(r_{c}\right)^{2}, \\
\dot{\epsilon}_{r \theta}^{-} & =\frac{1}{2 \eta}\left(1-\frac{\tau_{Y}}{\left|\tau_{d}^{-}\right|}\right) \tau_{r \theta}\left(r_{c}\right),
\end{aligned}
$$

with now

$$
\left|\boldsymbol{\tau}_{d}^{-}\right|=\left(2 \tau_{r \theta}\left(r_{c}\right)^{2}+2 \frac{\lambda^{2}}{\eta^{2}} \tau_{r \theta}\left(r_{c}\right)^{4}\right)^{1 / 2} .
$$

As $\dot{\epsilon}_{r \theta}^{-} \neq \dot{\epsilon}_{r \theta}^{+}$, the strain rate is discontinuous at $r=r_{c}$ and we can define a critical strain rate

$$
\dot{\varepsilon}_{r \theta}^{c}=\frac{1}{2 \eta}\left[1-\frac{\tau_{Y}}{\sqrt{2}\left|\tau_{r \theta}\left(r_{c}\right)\right|} \frac{1}{\left(1+\frac{\lambda^{2}}{\eta^{2}} \tau_{r \theta}\left(r_{c}\right)^{2}\right)^{1 / 2}}\right] \tau_{r \theta}\left(r_{c}\right) .
$$

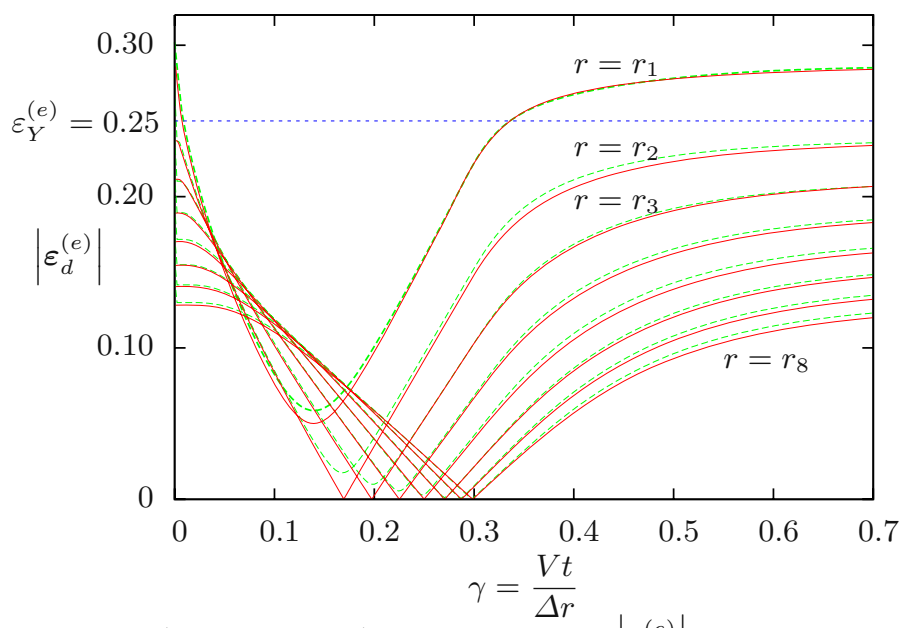

Fig. 7. (Colour online) Transient case: $\left|\varepsilon_{d}^{(e)}\right|$ versus $t$ for $r$ from $r=r_{1}$ to $r_{8}$. Dashed green lines: former computations; solid red lines: present computations.

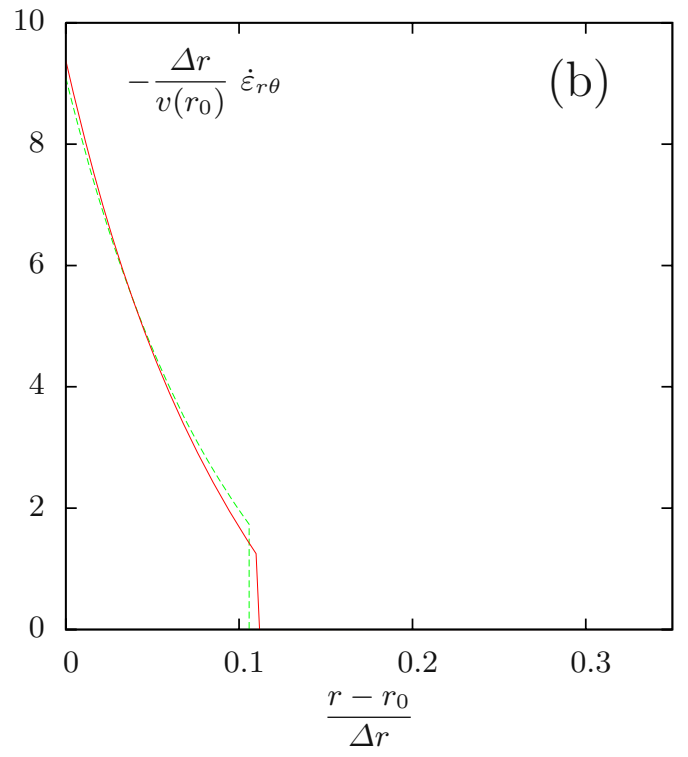

Fig. 8. (Colour online) Stationary case: (b) Shear strain rate $\dot{\varepsilon}_{r \theta}$ versus $r$. There is no experimental data available for the comparison. Dashed green lines: former computations; solid red lines: present computations.

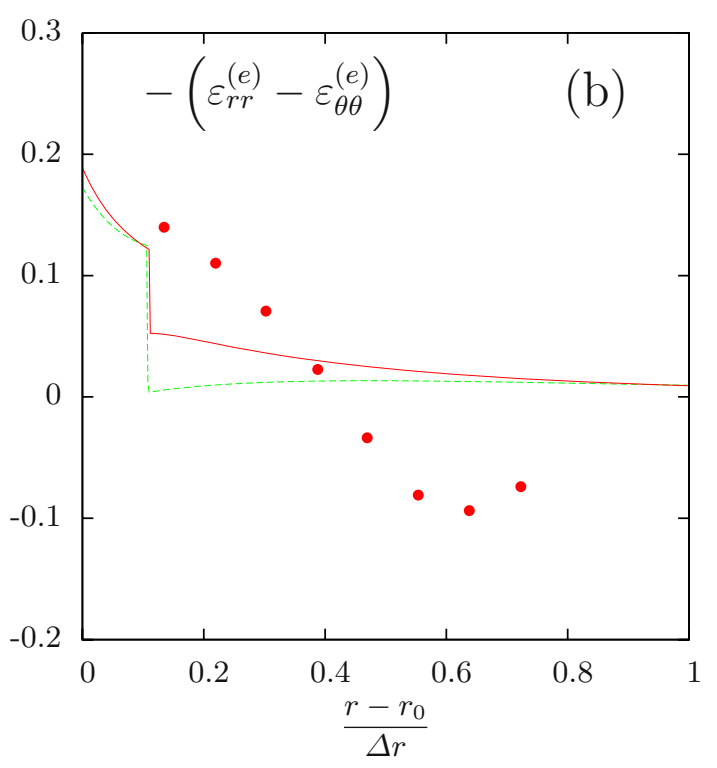

Fig. 9. (Colour online) Stationary case: (b) Difference of normal components. $-\left(\varepsilon_{r r}^{(e)}-\varepsilon_{\theta \theta}^{(e)}\right)$ versus $r$. Dashed green lines: former computations; solid red lines: present computations; -: experimental data.

\section{References}

1. I. Cheddadi, P. Saramito, C. Raufaste, P. Marmottant, F. Graner, Eur. Phys. J. E 27, 123 (2008). 